\section{Case Reports in Oncology}

\title{
A Shock-Like Pain and Inability to Ambulate: Thoracic Spinal Cord Compression from Hepatocellular Carcinoma
}

\author{
Kay Chen ${ }^{a}$ Mashya Abbassi ${ }^{b}$ Naomi Y. Ko ${ }^{c}$ \\ aDepartment of Medicine, Boston Medical Center, Boston, MA, USA; bepartment of \\ Radiology, Boston Medical Center, Boston, MA, USA; 'Department of Medicine, Section of \\ Hematology Oncology, Boston University and Boston Medical Center, Boston, MA, USA
}

Keywords

Hepatocellular carcinoma · Oncological emergency · Cord compression

\begin{abstract}
Hepatocellular carcinoma commonly metastasizes to organs, but there are few reports of vertebral metastases causing cord compression. Here, we present a case of thoracic cord compression in a patient with advanced hepatocellular carcinoma. Providers' and patient's awareness of this risk is important, as this is an oncological emergency.
\end{abstract}

(C) 2021 The Author(s).

Published by S. Karger AG, Basel

\section{Introduction}

In the United States, hepatocellular carcinoma (HCC) is the ninth leading cause of cancerrelated deaths with a rising incidence and mortality. It is usually diagnosed late, with a 2-year survival of less than $50 \%$ and arises in patients with chronic liver disease such as hepatitis B or hepatitis $\mathrm{C}$ [1]. The most common locations for extrahepatic metastases are the lungs, lymph nodes, bones, and adrenal glands. Bone metastases are thought to occur in roughly $28 \%$ of people compared to lung or lymph node metastases, which are seen in greater than $50 \%$ of patients [2]. Here, we report a case of a cord compression in a patient with back pain and advanced HCC. 


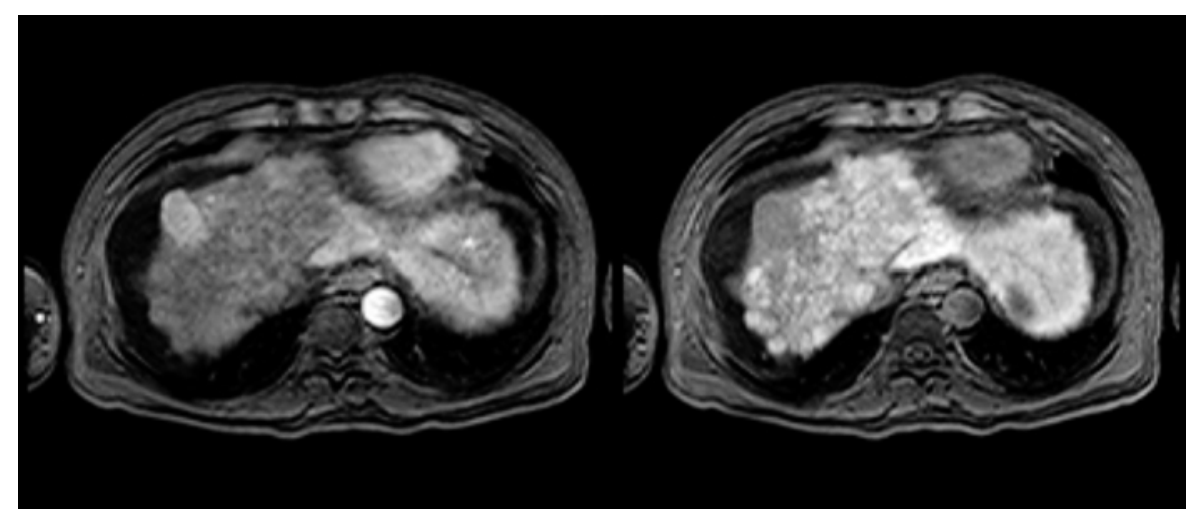

Fig. 1. MRI of the liver. Axial T1 imaging with contrast. There is a $3.2 \times 2.1 \mathrm{~cm}$ arterially enhancing lesion in segment IVa with washout on delayed imaging. There is a second arterially enhancing lesion with washout measuring $1.3 \times 1.0 \mathrm{~cm}$ in segment IV not seen on these images. There are several adjacent subcentimeter nodules of enhancement in the medial aspect of segment IVa. Additional findings include cirrhosis, hepatosplenomegaly, and ascites.

\section{Case Report}

A 56-year-old Vietnamese male with a past medical history notable for Child-Pugh Class B cirrhosis with relatively preserved liver synthetic function and HCC with multiple liver lesions, likely secondary to chronic hepatitis C (for which he completed treatment), hepatitis $\mathrm{B}$, and previous alcohol use disorder presented with an inability to walk for 3 days. The patient reported he had not been in his usual state of health for the last 2 months. He was more fatigued, with increasing abdominal pain and new back pain. His functional status declined in that he was spending $>50 \%$ of his day in bed and he was no longer independent in his activities of daily living. He continued to present every 1-2 weeks for therapeutic paracenteses, which he had been requiring for about 6 months. He then acutely developed worsening back pain with shock-like sensations that radiated down his bilateral lower extremities and a sudden inability to stand. He also had numbness and tingling bilaterally which started 1-2 inches above the umbilicus. He also reported having to strain to urinate.

Regarding his cancer, he was diagnosed in December 2016, 14 months prior to his presentation. He had undergone regular screening liver ultrasounds, which were unremarkable. However, he was noted to have a slowly rising alpha-fetoprotein level for a year prior to diagnosis to a level of $1,248.4 \mathrm{ng} / \mathrm{mL}$ and a high suspicion for HCC; an MRI of the liver with contrast was obtained, which showed 2 lesions, measuring up to $3.2 \mathrm{~cm}$ (shown in Fig. 1). He underwent transarterial chemoembolization in February 2017 and March 2017. Although his alpha-fetoprotein rose to 4,313.6 mg/mL, a repeat MRI in April 2017 showed no suspicious residual cancer. He was monitored with serial MRIs of the liver, and found to have recurrence in July 2017 and a third transarterial chemoembolization in September 2017. His alpha-fetoprotein at that point was $>20,000 \mathrm{ng} / \mathrm{mL}$, suggesting advanced disease. Progression was detected again via repeat liver MRI in December 2017.

On this admission, he initially presented to an outside hospital emergency room where a CT scan revealed lesions in T6-T9 vertebral bodies with epidural extension and cord compression at the T8-T9 level. He was then transferred to our hospital for neurosurgical evaluation. He was hemodynamically stable. His physical exam was notable for abdominal distension and tenderness with scattered petechiae, and 5/5 strength in the upper extrem- 


\section{Case Reports in Oncology}

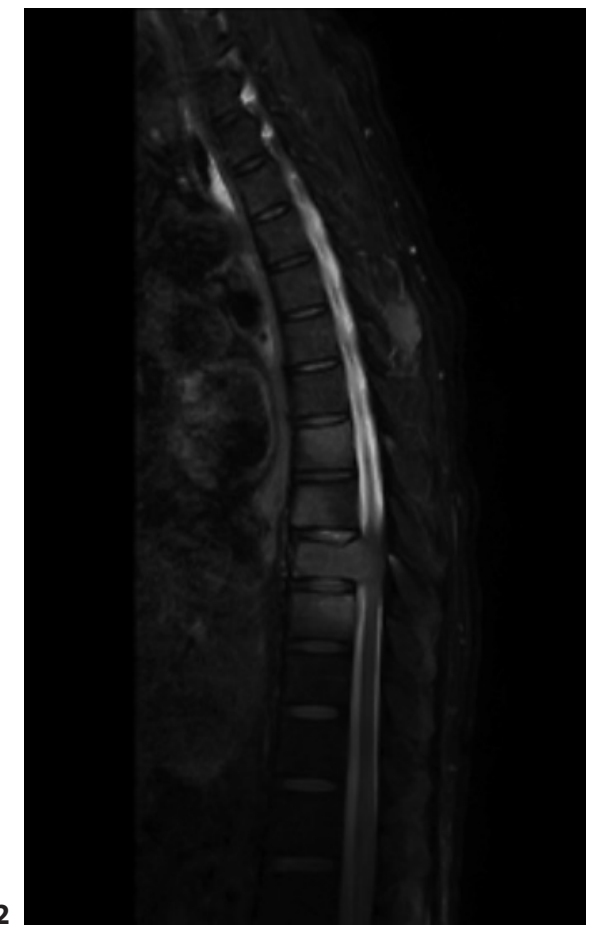

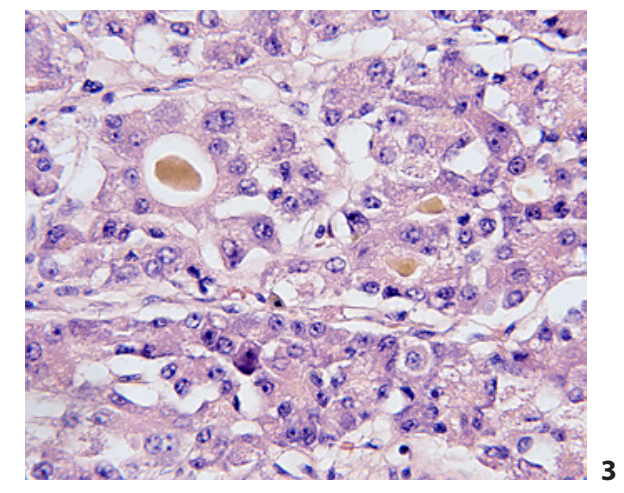

Fig. 2. MRI of the spine. T2 STIR sagittal imaging. Compression deformity of T8 with posterior propulsion of the vertebral body causing narrowing of the spinal canal and impingement of the thecal sac. There is abnormal signal and swelling of the spinal cord at this level. There are multiple areas of abnormal T2 signal including the vertebral bodies of T6, T7, T9, and the spinous process of $\mathrm{T} 3$, all of which are concerning for metastatic disease.

Fig. 3. Pathology slide. Histological examination of the T8 tumor with hematoxylin and eosin staining.

ities, and $4 / 5$ strength in the lower extremities. His laboratory studies were largely at baseline with a sodium of $133 \mathrm{mmol} / \mathrm{L}$, creatinine of $2.09 \mathrm{mg} / \mathrm{dL}$, INR of 1.02, AST of $88 \mathrm{U} / \mathrm{L}$, ALT of $64 \mathrm{U} / \mathrm{L}$, and ALP of $298 \mathrm{U} / \mathrm{L}$.

His spine MRI showed a compression deformity and enhancement of T8 with posterior propulsion of the vertebral body, causing impingement of the spinal canal with swelling and abnormal spinal cord signal (shown in Fig. 2). He also had lesions in the T3, T6, T7, T9, and S2 concerning for metastatic disease. Histology of the T8 lesion revealed polygonal cells typical of hepatocyte architecture. However, these cells contained prominent nucleoli and gland-like structures, which are abnormal features that confirmed the diagnosis of metastatic HCC (shown in Fig. 3).

He was admitted and given IV dexamethasone 10 mg once followed by 4 mg every $6 \mathrm{~h}$, with some improvement in his weakness. Neurosurgery and radiation oncology evaluated the patient, and planned for surgery followed by radiation. After platelet transfusions for a goal count of 100,000 or greater, he underwent T7-T9 laminectomy, T8 costotransversectomy and corpectomy for tumor resection, cage reconstruction, T5-T11 fusion with allograft, and vertebral augmentation cementoplasty.

Postoperatively, his course was complicated by toxic metabolic encephalopathy and multiorgan dysfunction. He developed a portal vein thrombus, bradycardia from metabolic derangements, worsening renal and liver function, a leukocytosis with an unknown source of infection, and hypoxia. Ultimately, he was intubated and transferred to the ICU after a seizure. The health care proxy and family made the decision to terminally extubate the patient rather than continue with aggressive measures, and the patient passed away. 


\section{Discussion}

HCC is an aggressive primary liver cancer that develops largely as a result of chronic inflammation [3]. As mentioned, metastasis to the bones is not common, but when it does occur, the lumbar and thoracic spine, ribs, long bones, and pelvis are most commonly involved [2]. Cord compression is an uncommon manifestation of all malignancies, with one study estimating the average annual incidence of malignancy-associated cord compression in cancer patients to be $3.4 \%$ in the United States [4]. Cord compression due to HCC is rare. When discovered, it is an oncological emergency due to the potential for permanent loss of neurological function with treatment delays. The outcome largely depends on the patient's neurological function prior to any definitive treatment [5].

The hematogenous dissemination of this cancer is thought to occur when the tumor invades into the hepatic vein and travels to the vertebral venous plexus [6]. In the setting of portal hypertension, the collaterals formed between the portal, abdominal, and pelvic venous system lead to lesions in the lower thoracic and lumbar spine [7]. Radiographically, the metastases are found in the vertebral body $74 \%$ of the time and the vertebral appendix $52 \%$ of the time. The bony lesions are often osteolytic lesions, although pure osteoblastic and mixed lesions have been seen as well. Soft tissue masses can also form in within the bony lesion or the paravertebral space [8].

Treatment includes steroids as a bridge to surgery and/or radiation therapy. The dosage of steroids is controversial as higher initial dosages are associated with adverse effects such as a perforated gastric ulcer, psychosis, and infection [9]. In addition, surgery is not insignificant, as one study reported an $86 \%$ morbidity rate with lumbar surgery in patients who are Childs Pugh Class B, like this patient. This morbidity rate is likely similar with thoracic spinal surgery [10].

In this particular case, the patient had multiple risk factors for HCC, including prior alcohol use, hepatitis B, and hepatitis C, which likely contributed to a more severe picture. He was unlikely to be ambulatory in the end, given he was non-ambulatory for nearly $72 \mathrm{~h}$ preceding his presentation. Intriguingly, while the patient did complain of new back pain, he also endorsed worsening chronic abdominal pain which he had attributed to his tense ascites. Thoracic radiculopathy can present with a band-like abdominal pain, so it is important to tease out any change in the characteristics of the pain [11].

There are a few case reports that describe cord compression from HCC, detailing lesions in the cervical, thoracic, and lumbar spine $[12,13]$. Most describe the classic presentation of back pain and new neurological deficits in the setting of known HCC. In some of the case reports, the patient had no known diagnosis of cancer $[12,13]$. The majority of cases described patients with chronic hepatitis B leading to HCC. In fact, patients with HCC as a result of hepatitis B infection were found to have a higher incidence of bony metastasis [14], likely because they have larger tumors $(>5 \mathrm{~cm})$ at the time of diagnosis. This may also be because $40 \%$ of patients with chronic hepatitis B do not develop cirrhosis prior to HCC. These patients therefore do not undergo routine surveillance practices and are diagnosed at later stages [15].

Overall, we present a case of cord compression due to advanced HCC. While rare, it is likely that the incidence of spinal cord compression in HCC will increase as mean survival increases. One might have a lower threshold to consider spinal imaging in the setting of advanced HCC.

\section{Karger'}




\section{Acknowledgements}

We thank Dr. Andrey Prilutskiy at Boston Medical Center for his assistance with the pathology slides.

\section{Statement of Ethics}

Consent for publication of this case report including publication of images was obtained from the patient's health care proxy.

\section{Conflict of Interest Statement}

The authors have no conflict of interest to report.

\section{Funding Sources}

None.

\section{Author Contributions}

Dr. Chen was responsible for literature review and writing the manuscript. Dr. Abbassi was responsible for generating figures and captions. Dr. Ko edited and commented for the final draft.

\section{References}

1 Golabi P, Fazel S, Otgonsuren M, Sayiner M, Locklear CT, Younossi ZM. Mortality assessment of patients with hepatocellular carcinoma according to underlying disease and treatment modalities. Medicine (Baltimore). 2017;96(9):e5904.

2 Becker AK, Tso DK, Harris AC, Malfair D, Chang SD. Extrahepatic metastases of hepatocellular carcinoma: A spectrum of imaging findings. Can Assoc Radiol J. 2014;65(1):60-6.

3 Coskun M. Hepatocellular carcinoma in the cirrhotic liver: evaluation using computed tomography and magnetic resonance imaging. Exp Clin Transplant. 2017;15(Suppl 2):36-44.

4 Mak KS, Lee LK, Mak RH, Wang S, Pile-Spellman J, Abrahm JL, et al. Incidence and treatment patterns in hospitalizations for malignant spinal cord compression in the United States, 1998-2006. Int J Radiat Oncol Biol Phys. 2011;80(3):824-31.

5 Bach F, Larsen BH, Rohde K, Børgesen SE, Gjerris F, Bøge-Rasmussen T, etal. Metastatic spinal cord compression. Occurrence, symptoms, clinical presentations and prognosis in 398 patients with spinal cord compression. Acta Neurochir (Wien). 1990;107(1-2):37-43.

6 Okazaki N, Yoshino M, Yoshida T, Hirohashi S, Kishi K, Shimosato Y. Bone metastasis in hepatocellular carcinoma. Cancer. 1985;55(9):1991-4.

7 Fukutomi M, Yokota M, Chuman H, Harada H, Zaitsu Y, Funakoshi A, et al. Increased incidence of bone metastases in hepatocellular carcinoma. Eur J Gastroenterol Hepatol. 2001;13(9):1083-8.

8 Chen HY, Ma XM, Bai YR. Radiographic characteristics of bone metastases from hepatocellular carcinoma. Contemp Oncol (Pozn), 2012. 16(5):424-31.

9 George R, Jeba J, Ramkumar G, Chacko AG, Leng M, Tharyan P. Interventions for the treatment of metastatic extradural spinal cord compression in adults. Cochrane Database Syst Rev. 2015;2015(4):Cd006716.

10 Liao JC, Chen WJ, Chen LH, Niu CC, Fu TS, Lai PL, et al. Complications associated with instrumented lumbar surgery in patients with liver cirrhosis: a matched cohort analysis. Spine J. 2013;13(8):908-13.

11 Choi HE, Shin MH, Jo GY, Kim JY. Thoracic radiculopathy due to rare causes. Ann Rehabil Med. 2016;40(3): 534-9. 
12 Nangolo HT, Roberto L, Segamwenge IL, Voigt A, Kidaaga F. Spinal cord compression: an unusual presentation of hepatocellular carcinoma. Pan Afr Med J. 2014;19:363.

13 Doval DC, Bhatia K, Vaid AK, Pavithran K, Sharma JB, Hazarika D, et al. Spinal cord compression secondary to bone metastases from hepatocellular carcinoma. World J Gastroenterol. 2006;12(32):5247-52.

14 Harding JJ, Abu-Zeinah G, Chou JF, Owen DH, Ly M, Lowery MA, et al. Frequency, morbidity, and mortality of bone metastases in advanced hepatocellular carcinoma. J Natl Compr Canc Netw. 2018;16(1):50-8.

15 Hiotis SP, Rahbari NN, Villanueva GA, Klegar E, Luan W, Wang Q, et al. Hepatitis B vs. hepatitis C infection on viral hepatitis-associated hepatocellular carcinoma. BMC Gastroenterol. 2012;12:64. 